\title{
Efecto gastroprotector y capacidad antioxidante del extracto acuoso de las vainas de Caesalpinia spinosa 'tara', en animales de experimentación
}

\section{Gastroprotective effect and antioxidant capacity of the aqueous extract of the pods of Caesalpinia spinosa "tara" in experimental animals}

\author{
Rosalía Callohuari ${ }^{1, a}$, Miguel Sandoval Vegas ${ }^{1, b}$, Oscar Huamán Gutiérrez ${ }^{1, b}$ \\ ${ }^{1}$ Facultad de Medicina, Universidad Nacional Mayor de San Marcos, Lima, Perú. \\ ${ }^{a}$ Egresada de la Maestría en Bioquímica; b Centro de Investigación de Bioquímica y Nutrición.
}

\section{Correspondencia}

Miguel Sandoval Vegas mhsave@gmail.com

Recibido, 14 junio 2016

Evaluado, 28 setiembre 2016

Aceptado, 8 octubre 2016

El contenido del manuscrito no ha sido publicado previamente.

Fuente de financiamiento:

Autofinanciado, con el apoyo

de las instalaciones, equipos $y$ algunos reactivos del Centro de Investigación de Bioquímica y Nutrición "Alberto Guzmán Barrón" de la Facultad de Medicina de la UNMSM.

Ningún conflicto de interes.

Citar como: Callohuari R, Sandoval Vegas M, Huamán Gutiérrez O. Efecto gastroprotector y capacidad antioxidante del extracto acuoso de las vainas de Caesalpinia spinosa 'tara', en animales de experimentación. An Fac med. 2017;78(1):61-6. DOI: http://dx.doi. org/10.15381/anales.v78i1.13023
An Fac med. 2017;78(1):61-6 / http://dx.doi.org/10.15381/anales.v78i1.13023

Resumen

Objetivo. Determinar el efecto protector del extracto acuoso de las vainas de tara Caesalpinia spinosa (Molina) Kuntze, en la mucosa gástrica de animales de experimentación. Diseño. Experimental. Institución. Centro de Investigación de Bioquímica y Nutrición, Facultad de Medicina, Universidad Nacional Mayor de San Marcos, Lima, Perú. Material biológico. Extracto acuoso de las vainas de tara (EAVT) y 36 ratas albinas macho $(217 \mathrm{~g} \pm$ $22 \mathrm{~g}$ ). Intervenciones. Las ratas albinas previo ayuno de $24 \mathrm{~h}$ fueron divididas en 6 grupos: $\mathrm{Gl}$ y $\mathrm{Gll}, \mathrm{NaCl}$ 0,9\% a $20 \mathrm{~mL} / \mathrm{kg}$; GIII, GIV y GV, EAVT en dosis de 100, 400 y $800 \mathrm{mg} / \mathrm{kg}$, respectivamente; y, GVI hidroflat 500 $\mathrm{mg} / \mathrm{kg}$. Una hora después, se provocó la injuria gástrica con etanol $70^{\circ}$ para luego realizar la gastrectomía. Principales medidas de resultados. Porcentaje de protección de la mucosa, empleando el software ImageJ $1.48 \mathrm{v}$, y para la actividad antioxidante el método 2,2-difenil-1-picrilhidracil (DPPH). Resultados. El EAVT presentó compuestos fenólicos y taninos en mayor cantidad. Exhibió alta actividad antioxidante $\left(\mathrm{IC}_{50}=1,12\right.$ $\pm 0,04 \mu \mathrm{g} / \mathrm{mL})$. El mayor porcentaje de protección se observó a las dosis de $800 \mathrm{mg} / \mathrm{kg}(99,7 \%)$ y $400 \mathrm{mg} /$ $\mathrm{kg}(73,1 \%) p<0,01$, lo cual fue confirmado por el análisis histopatológico. Conclusiones. El EAVT mostró actividad antioxidante y protectora en el modelo experimental de lesión gástrica inducida por etanol de $70^{\circ}$, de manera dosis dependiente.

Palabras clave. Caesalpinia spinosa; Mucosa Gástrica; Gastritis; Mucoprotección; Tara.

Abstract

Objective: To determine the protective effect of the aqueous extract of the pods of Caesalpinia spinosa (Molina) Kuntze "tara" in the gastric mucosa of experimental animals. Design: Experimental. Institution: Research Center on Biochemistry and Nutrition, Faculty of Medicine, Universidad Nacional Mayor de San Marcos, Lima, Peru. Biological material: Aqueous extract tara pods (AETP) and 36 albino male rats $(217 \mathrm{~g} \pm 22$ g). Interventions: After a 24-hour fast, the albino rats were divided into 6 groups: $\mathrm{Gl}$ and $\mathrm{GII}, \mathrm{NaCl} 0.9 \%$ to 20 $\mathrm{mL} / \mathrm{kg}$; GIII, GIV and GV, AETP at doses of 100,400 and $800 \mathrm{mg} / \mathrm{kg}$ respectively; and GVI, Hidroflat $500 \mathrm{mg} / \mathrm{kg}$. An hour later, gastric injury was caused with $70^{\circ}$ ethanol and then gastrectomy was performed. Main outcome measures: Percentage of mucosal protection, using the software ImageJ $1.48 \mathrm{v}$, and the antioxidant activity method 2, 2-diphenyl-1-picrylhydrazyl (DPPH). Results: AETP presented tannins and phenolic compounds in large amounts. High antioxidant activity $\left(\mathrm{IC}_{50}=1,1 \pm 0.04 \mu \mathrm{g} / \mathrm{mL}\right)$ was exhibited. The highest percentage of protection was observed at doses of $800 \mathrm{mg} / \mathrm{kg}(99.7 \%)$ and $400 \mathrm{mg} / \mathrm{kg}(73.1 \%), \mathrm{p}<0,01$, confirmed by histopathological analysis. Conclusions: AETP exhibited antioxidant and protective activity in the experimental model of gastric injury induced by ethanol $70^{\circ}$, in a dose-dependent manner.

Keywords: Caesalpinia spinosa; Gastric Mucosa; Gastritis; Mucus Protection; Tara. 


\section{INTRODUCCIÓN}

La tara es una leguminosa nativa de Sudamérica, de la cual las vainas han sido ampliamente utilizadas en la medicina tradicional para tratar diversas dolencias debido a su efecto antibiótico contra enfermedades respiratorias e infecciones de la piel. El contenido de taninos en las vainas de la tara representa el $40 \%$ al $60 \%$ de su peso seco ${ }^{(1)}$. Entre las propiedades atribuidas a los taninos de la tara, está su carácter astringente, antiinflamatorio, antimicrobiano y antiséptico, además de ser empleada para tratar úlceras y ayudar a la cicatrización (2).

Las propiedades físico-químicas (actividad antioxidante, polifenoles totales y contenido de taninos) de extractos, obtenidos por maceración en diferentes solventes y soxhlet, de las vainas de tara han sido reportados en estudios previos, en especial los extractos acuosos, indicando una buena actividad antioxidante de acuerdo a los métodos más utilizados, como el ABTS (ácido 2,2'-azino-bis3-etilbenzotiazolin-6-sulfónico) y DPPH (1,1-difenil-2-picril-hidrazilo) ${ }^{(3-5)}$.

Por otro lado, la gastritis y úlceras pépticas son enfermedades muy frecuentes en nuestra población. La gastritis ocurre en el contexto de una enfermedad sistémica grave, como traumatismos, quemaduras, sepsis, insuficiencia hepática y renal y shock, pero comúnmente es debida a la injuria directa de la mucosa por agentes tales como fármacos antiinflamatorios no esteroideos (AINE), etanol, esteroides (dosis altas), agentes alcalinos y ácidos fuertes. El tratamiento agudo con etanol produce lesiones y erosiones de la mucosa gástrica, aumentando los niveles de peroxidación lipídica, daño del ADN y reduciendo el contenido de glutatión reducido (GSH) en la mucosa gástrica de rata. El daño gástrico por etanol también se debe a su acción vasoconstrictora de las venas y arterias de la mucosa gástrica, produciendo la congestión, inflamación y daño tisular ${ }^{(6,7)}$. Cuadros menos graves se presentan en pacientes bajo tensiones emocionales ${ }^{(8)}$.

Se han comunicado las acciones de gastroprotección y antiulcerosa de varios productos vegetales, en diferentes mo- delos experimentales, y en estos estudios se señala que entre los componentes se encuentran los taninos, atribuyéndole tales propiedades y su capacidad antioxidante, los cuales pueden constituir la base molecular del efecto antiinflamatorio que ejercen muchas sustancias naturales y sintéticas ${ }^{(9)}$. Asimismo, se están reevaluando nuevos métodos de estudio de las plantas medicinales, ya sea a nivel de extracción, análisis, formulación y dosificación, en diversos modelos animales. También se están ensayando compuestos activos aislados, los cuales tienen una actividad muy diferente al extracto total inicial.

La importancia de la presente investigación es brindar un aporte al estudio fitoquímico y farmacológico de la especie vegetal C. spinosa 'tara', a la que se atribuye tradicionalmente muchas propiedades medicinales, entre las cuales se señala de manera verbal el protector de la mucosa del estómago, probablemente por la capacidad antioxidante que presentan sus componentes. Es por ello que el objetivo principal fue determinar la actividad protectora y antioxidante del EAVT Caesalpinia spinosa (Molina) Kuntze frente a la injuria aguda de la mucosa gástrica inducida por etanol, en ratas albinas.

\section{MÉTODOS}

Se realizó un estudio de diseño experimental aleatorizado con grupo control en animales de experimentación.

Las vainas de Caesalpinia spinosa 'tara', fueron adquiridas del fundo Mostacero, ubicado en el Sector de Magollo, a 150 m.s.n.m de la provincia, departamento de Tacna.

La especie fue identificada por el herbario Takana de la Universidad Nacional Jorge Basadre Grohmann de la ciudad de Tacna. Las vainas fueron limpiadas con escobilla de cerdas finas, secadas y extraídas las semillas; las vainas fueron molidas utilizando un molino de cuchillas eléctrico (Willey Mill St. Modell $N^{\circ} 3$ ) y tamizadas hasta obtener un polvo fino. Se realizó la extracción acuosa con agua destilada $(1: 4 \mathrm{p} / \mathrm{v})$ a $65^{\circ} \mathrm{C}$ en un baño ma- ría por una hora. El extracto fue filtrado a través de varias capas de gasa, luego a través de papel de filtro № 40 al vacío; el filtrado fue concentrado en una estufa de aire circulante de $40^{\circ} \mathrm{C}$ por 72 horas, para obtener lo que significa EAVT seco. Finalmente se almacenó en frascos de color ámbar y en refrigeración a 4으 C hasta su uso.

El análisis fitoquímico de los constituyentes del EAVT fue basado en la aplicación de pruebas de coloración y precipitación, de acuerdo al método descrito por Lock $\mathrm{O}^{(10)}$.

Para la observación de la capacidad antioxidante del EAVT se utilizó una modificación del método 2,2-difenil-1-picrilhidracil (DPPH), descrito por Yamaguchi y col, $1998^{(11)}$.

EI DPPH fue disuelto en metanol a una concentración de $20 \mu \mathrm{g} / \mathrm{mL}$; se preparó inmediatamente antes de ser utilizado. El estándar usado fue el ácido ascórbico diluido a una concentración de $10 \mu \mathrm{g} / \mathrm{mL}$. Ambas soluciones fueron protegidas de la luz. El EAVT fue disuelto a una concentración de $8 \mu \mathrm{g} / \mathrm{mL}$ en solución acuosa. En tubos se colocaron $200 \mu \mathrm{L}$ de las diluciones del extracto o de ácido ascórbico, a los cuales se les adicionó $800 \mu \mathrm{L}$ de solución de DPPH. La solución resultante fue agitada en un vórtex por $10 \mathrm{~s}$ e incubada a temperatura ambiente por $15 \mathrm{~min}$. La disminución de la absorbancia fue leída contra el blanco en el espectrofotómetro a 517 nm. La capacidad de captación de radicales del EAVT fue expresado como porcentaje (\%) de inhibición, determinado por la siguiente expresión:

$\%$ inhibición $=\frac{\text { (Abs control }- \text { Abs muestra) }}{\text { Abs control }} \times 100$

Donde, Abs control es la absorbancia a tiempo cero y Abs muestra es la absorbancia a los 15 minutos. Todos los ensayos se realizaron por triplicado y se promediaron los resultados. El cálculo correspondiente al valor de $\mathrm{IC}_{50}$, se determinó mediante un análisis de regresión de los porcentajes de inhibición con la concentración necesaria de los extractos, para inhibir el 50\% del radical DPPH.

La capacidad protectora se realizó a través de un experimento in vivo en 36 ratas albinas machos Rattus norvegicus 
Tabla 1. Resultados del estudio fitoquímico del EAVT Caesalpinia spinosa.

\begin{tabular}{ccc} 
Ensayos & Metabolitos & Resultado \\
R. Gelatina & Taninos & + \\
R. Tricloruro férrico & Compuestos fenólicos & + \\
R. Dragendorff & Alcaloides & - \\
R. Mayer & Alcaloides & - \\
R. NaOH 5\% & Cumarinas & - \\
R. Molish & Glicósidos & - \\
R. Shinoda & Flavonoides & + \\
\hline
\end{tabular}

(+) Presencia, (-) Ausencia.

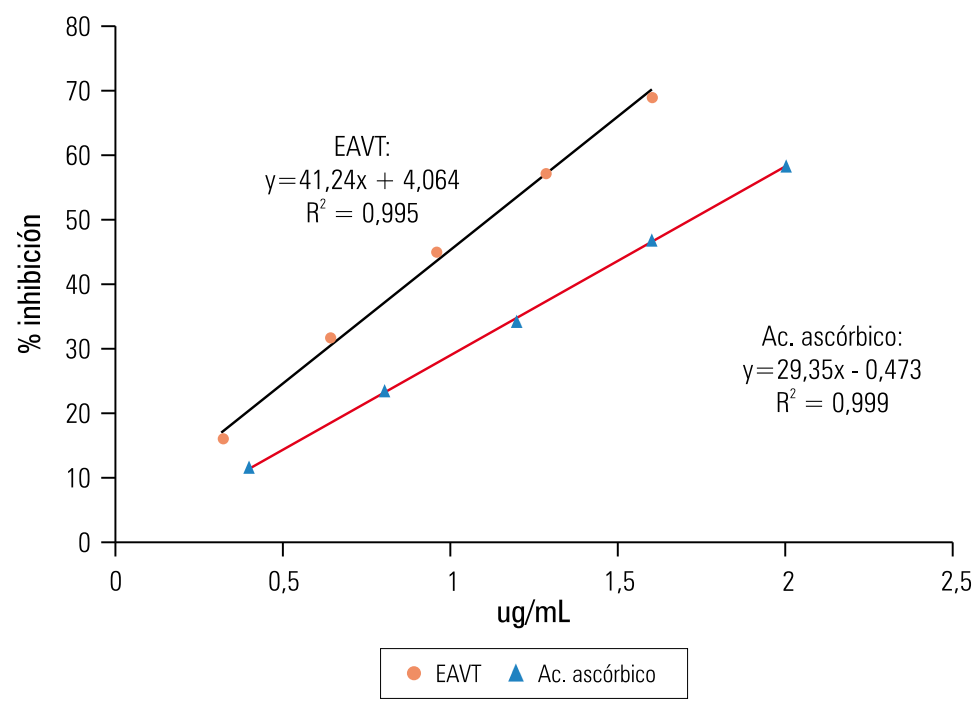

Figura 1. Capacidad antioxidante del extracto EAVT y vitamina C frente al radical DPPH. cepa Holtzman (217 g $\pm 22 \mathrm{~g}$ ) adquiridos del bioterio de la Universidad Nacional Agraria La Molina (UNALM). Fueron mantenidas en ayuno sólido de 24 h. Los animales se dividieron aleatoriamente en 6 grupos (G), a los cuales se administró mediante canulación orogástrica en dosis única las siguientes sustancias: grupo I solución $\mathrm{NaCl}$ 0,9\% a $20 \mathrm{~mL} / \mathrm{kg}$; grupo II IV y $\vee$ el EAVT en dosis de 100 mg/kg, 400 $\mathrm{mg} / \mathrm{kg}$ y $800 \mathrm{mg} / \mathrm{kg}$, respectivamente; y grupo VI, hidroflat $500 \mathrm{mg} / \mathrm{kg}$. Transcurrida $1 \mathrm{~h}$ de la administración, se provocó la lesión gástrica con etanol de $70^{\circ}{ }^{(12)} \mathrm{a}$ los grupos II al VI. Al grupo I se administró nuevamente solución $\mathrm{NaCl}$ 0,9\%, que correspondió al grupo control negativo o mucosa gástrica. Posteriormente, trascusolución $\mathrm{NaCl}$ 0,9\% a $20 \mathrm{~mL} / \mathrm{kg}$; grupos III, basal, que muestra el estado natural de la vatura mayor. Para el análisis macroscópico de los estómagos, se obtuvieron las imágenes digitalizadas y se midió del área de lesión gástrica en $\mathrm{mm}^{2}$ por medio del software Images del National Institute of Health (NIH) ${ }^{(13)}$. El cálculo del porcentaje de protección de la mucosa gástrica fue expresado según Sandoval y col. ${ }^{(14)}$

Se realizó también el estudio microscópico, para lo cual cada tejido gástrico fue conservado en formol al $10 \%$ para su estudio histopatológico. El tratamiento de las muestras y la lectura de las láminas fueron realizadas en el Instituto de Patología y Biología Molecular Arias-Stella.

Para el análisis del efecto protector, los resultados fueron expresados como media $\pm D E(n=6)$ y se procesaron por la prueba de Kruskal-Wallis seguida de la prueba de U Mann-Whitney U. La significancia estadística fue considerada como $p<0,05$, con intervalo de confianza del 95\%. La IC50 se determinó mediante regresión lineal.

\section{RESULTADOS}

Al realizar el análisis fitoquímico del extracto acuoso de vainas de tara (EAVT), se evidenció la presencia de compuestos fenólicos, entre ellos taninos y flavonoides (tabla 1).

El EAVT tuvo un porcentaje de inhibición alto del radical DPPH comparado con el estándar ácido ascórbico, como se puede apreciar en la figura 1.

Asimismo, el EAVT presentó un $\mathrm{IC}_{50}=$ $1,12 \pm 0,04 \mu \mathrm{g} / \mathrm{mL}$, comparado con el es-
Tabla 2. Índice de lesión y porcentaje de protección de cada grupo experimental. Los datos son presentados como media $\pm \mathrm{DE}$.

\begin{tabular}{ccc} 
Grupo $(\mathrm{n}=6)$ & Promedio del área de lesión $\left(\mathrm{mm}^{2}\right)$ & \% Protección \\
Control positivo & $62,0 \pm 27,5$ & - \\
EAVT $100 \mathrm{mg} / \mathrm{kg}$ & $21,2 \pm 12,9 \mathrm{a}, \mathrm{c}$ & 65,7 \\
EAVT $400 \mathrm{mg} / \mathrm{kg}$ & $16,7 \pm 14,4^{\mathrm{b}, \mathrm{c}}$ & 73,1 \\
EAVT $800 \mathrm{mg} / \mathrm{kg}$ & $0,2 \pm 0,5^{\mathrm{b}, \mathrm{c}}$ & 99,7 \\
Hidroflat & $67,3 \pm 25,4^{\mathrm{Ns}}$ & $-8,6$ \\
\hline
\end{tabular}

Prueba de $U$ Mann-Whitney:

(a) $p<0,05$ comparado con el control: EAVT $100 \mathrm{mg} / \mathrm{kg} \mathrm{p}=0,016$.

(b) $p<0,01$ comparado con el control: EAVT $400 \mathrm{mg} / \mathrm{kg} p=0,004$; EAVT $800 \mathrm{mg} / \mathrm{kg} \mathrm{p}=0,003$.

(c) $p<0,01$ comparado con hidroflat: EAVT $100 \mathrm{mg} / \mathrm{kg} \mathrm{p}=0,006$; EAVT $400 \mathrm{mg} / \mathrm{kg} \mathrm{p}=0,004$; EAVT $800 \mathrm{mg} / \mathrm{kg} p=0,003$. (NS) no hubo significancia estadística con el control a $p<0,05$. 
tándar ácido ascórbico con $\mathrm{IC}_{50}=1,72 \pm$ $0,05 \mu \mathrm{g} / \mathrm{mL}$.

El efecto del tratamiento de la mucosa gástrica con el EAVT previo a la agresión con alcohol etílico a $70^{\circ}$, brindó protección a la mucosa de manera estadísticamente significativa mayor $(p<0,01)$ a las dosis más altas, comparadas con el control y el grupo con hidroflat (tabla 2). El EAVT a dosis de $800 \mathrm{mg} / \mathrm{kg}$ mostró un área de lesión de $0,2 \mathrm{~mm}^{2}$, representando un $99,7 \%$ de protección de la mucosa.

Las microfotografías de los cortes histológicos del grupo I mostraron una apariencia histológica de la mucosa gástrica normal, con superficie homogénea, arquitectura glandular conservada, lámina propia bien delimitada y vasos no congestionados (figura 3A). En contraste, el grupo control positivo presentó un amplio foco de erosión y necrosis de la mucosa superficial, profundidad de daño que comprometió más de la mitad de la mucosa y congestión vascular (figura 3B). El grupo que recibió el extracto $100 \mathrm{mg} /$ $\mathrm{kg}$ presentó pequeñas zonas de necrosis asociada a una anoxia provocada por el etanol y erosión leve superficial en algunos cortes y congestión de vasos (hema-

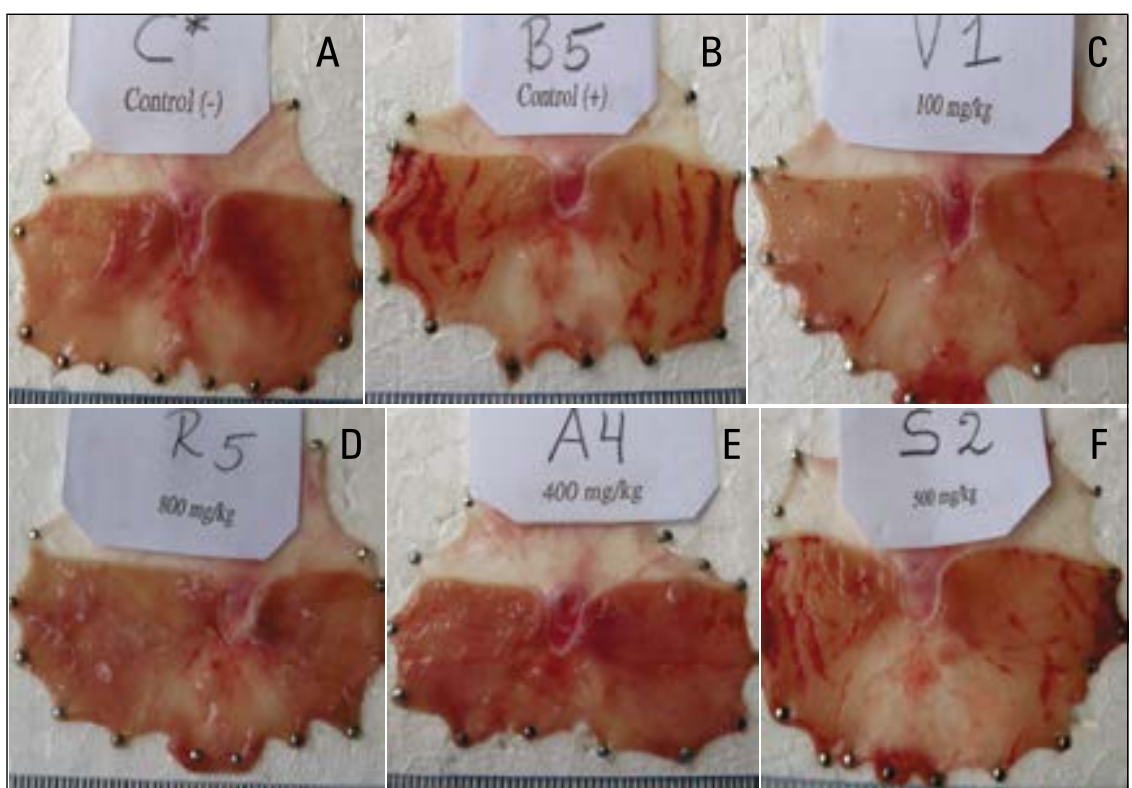

Figura 2. Aspecto macroscópico de la mucosa gástrica. Grupo control negativo y control positivo (A y B). Grupos del EAVT $100 \mathrm{mg} / \mathrm{kg}$ (C), $400 \mathrm{mg} / \mathrm{kg}$ (D) y $800 \mathrm{mg} / \mathrm{kg}$ (E) e hidroflat $500 \mathrm{mg} / \mathrm{kg}$ (F). en los métodos de preparación tradicionales, la mayoría de los remedios se preparan como extractos acuosos, evitando así los efectos tóxicos potenciales ${ }^{(16,17)}$, tal como se desarrolló la extracción en el presente estudio.

La capacidad de un extracto para eliminar los radicales libres se puede fundamentar en sus propiedades antioxidantes dados sus componentes y este mecanismo también podría ser una de las razones por las que el extracto ejerce su efecto protector gástrico del daño que causa el etanol. Así, la actividad antioxidante del EAVT fue determinada in vitro y comparada con un control positivo antioxidante, el ácido ascórbico. Los resultados obtenidos por el método del DPPH (figura 1) indican que el EAVT mostró un valor $I C_{50}$ mayor $\left(I C_{50}\right.$ de $\left.1,12 \mu \mathrm{g} / \mathrm{mL}\right)$ al estándar $\left(I_{50} \quad 1,72 \mu \mathrm{g} / \mathrm{mL}\right)$ para inhibir el radical DPPH. Dicha capacidad antioxidante se atribuiría al alto contenido de fenoles totales que presenta, esto de acuerdo con estudios previos que indican una relación directa entre actividad antioxidante y contenido de fenoles totales en hierbas, vegetales y frutos ${ }^{(18)}$, y esta a su vez con el contenido de taninos y su eficiencia antirradical $(19,20)$.

De acuerdo con la clasificación de Gomes de Melo y col. ${ }^{(21)}$, el EAVT muestra una buena actividad antioxidante, y resultados similares han sido reportados en otros estudios referentes a los extractos de vainas de tara ${ }^{(4,22)}$. La influencia del medio ambiente, nutrición y genotipo, así como el tiempo de la cosecha y su tratamiento post-cosecha pueden afectar la calidad y niveles de antioxidantes en las plantas. Así pues, el tipo de suelo y el pH tienen un efecto directo en la concentración de taninos ${ }^{(23,24)}$.

El modelo de lesión gástrica aguda por etanol a $70^{\circ}$ es útil para medir la actividad citoprotectora de una sustancia o elemento ${ }^{(12)}$. Para determinar la dosis efectiva del EAVT, se realizaron pruebas piloto y a partir de allí se establecieron tres concentraciones; 100,400 y 800 mg/ $\mathrm{kg}$. Los resultados mostrados en la tabla 2 indican que el EAVT presentó una relación de tipo dosis-efecto, ya que a las dosis de 400 y 800 mg/kg se obtuvo actividad protectora estadísticamente signi- 


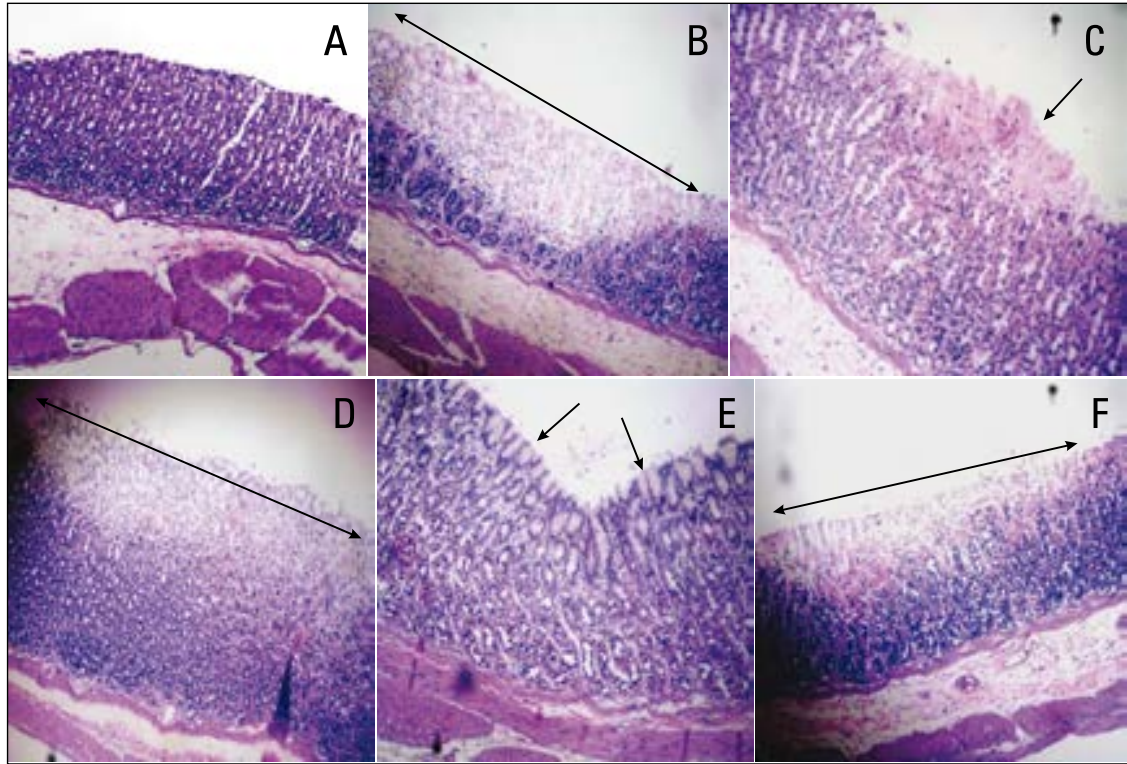

Figura 3. Aspecto microscópico de la mucosa gástrica (10x H-E). Grupo control negativo: apariencia histológica de la mucosa gástrica normal (A). Grupo control positivo: amplio foco de erosión y necrosis de la mucosa superficial $(\leftrightarrow)(B)$. Grupo del EAVT $100 \mathrm{mg} / \mathrm{kg}$ : necrosis pequeña y pérdida del epitelio $(\downarrow)(C)$. Grupo del EAVT $400 \mathrm{mg} / \mathrm{kg}$ : necrosis $(\leftrightarrow)(\mathrm{D})$. Grupo del EAVT 800

$\mathrm{mg} / \mathrm{kg}$ : erosión superficial mínima $(\downarrow)(E)$ y grupo hidroflat $500 \mathrm{mg} / \mathrm{kg}$ : necrosis (presencia de hematíes) $(\leftrightarrow)(F)$.

ficativa $(p<0,01)$, con una protección de 73,1 y $99,7 \%$, respectivamente. La dosis de $100 \mathrm{mg} / \mathrm{kg}$ tuvo actividad protectora menor $(65,8 \%)$, pero igualmente estadísticamente significativa comparado con el control $(p<0,05)$. Asimismo, cuando se comparó las tres dosis del EAVT con el antiácido hidroflat, estas tuvieron una diferencia estadísticamente significativa $(p<0,05)$.

Relaciones dosis-efecto similares a las referidas en el párrafo anterior, se han obtenido con fracciones de flavonoides ${ }^{(25)}$. Pero con fracciones de taninos se ha reportado una relación dosis-efecto inversa de tres dosis ensayadas de la fracción tanino obtenida por extracción metanólica, presentándose el mejor efecto en la gastroprotección a dosis de $25 \mathrm{mg} /$ $\mathrm{kg}$, que es cuatro veces menor que la dosis utilizada para el control positivo (carbenoxolona), atribuyéndole a los taninos la propiedad de precipitar microproteínas en el sitio de la úlcera, construyendo una película protectora que evita que las sustancias tóxicas puedan ser absorbidas y de esta manera promovería la resistencia a los agentes nocivos ${ }^{(26)}$.
En la figura 2 se puede observar el precipitado gelatinoso al realizar las observaciones macroscópicas de los estómagos tratados con dosis de $800 \mathrm{mg} / \mathrm{kg}$ y en menor cantidad a la dosis de 400 $\mathrm{mg} / \mathrm{kg}$. La hidrotalcita, un antiácido que contiene aluminio y magnesio similar al hidroflat, se ha comprobado que ejerce una acción protectora sobre la mucosa gástrica dañada en ratas y reduce significativamente la lesión inducida por etanol y AINEs mediante la preservación de las células endoteliales de los vasos sanguíneos de la mucosa y las células progenitoras epiteliales ${ }^{(27,28)}$.

Lo señalado anteriormente difiere con los resultados obtenidos en este estudio, ya que el hidroflat a la dosis usada no logró la protección mucogástrica deseada en comparación al extracto de tara. En otros estudios se ha demostrado que la administración oral de magnesio reduce el recuento de células parietales y aumenta las células mucosas de úlceras probable que el mecanismo por el cual el magnesio ejerce su propiedad antiulcerosa sea mediante la reducción de la masa inducidas por indometacina en ratas. Es celular parietal y estimulando la producción de la prostaglandina que estimula la producción de células mucosas ${ }^{(29)}$.

En un estudio reciente, acerca del efecto del pre tratamiento de la mucosa gástrica con el extracto alcohólico de vainas de $C$. spinosa ante lesiones en mucosa gástrica inducidas con ácido acetil salicílico en ratas holtzman, se observó que no presentó efecto protector a dosis de 100,200 y 300 mg/kg, atribuyéndole estos resultados al modelo de inducción de lesión utilizado, a la cepa holtzman que sería menos vulnerable al efecto del ácido acetil salicílico y, finalmente al tipo de taninos predominante en el extracto ${ }^{(5)}$, ya que los taninos están involucrados en la actividad antiulcerosa de diversas plantas medicinales.

En la evaluación histológica (figura 3), en nuestros resultados se observó que el grupo de animales pre tratados con el EAVT presentaron menor área de lesión en la superficie de la mucosa gástrica. El grupo control positivo tuvo ulceraciones hemorrágicas severas y agudas, inflamación y lesión petequial en la mucosa gástrica, amplios focos de erosión, necrosis y congestión vascular, sin infiltración de leucocitos, ni tejido de granulación y sin alterar la submucosa, similares al antiácido hidroflat. A dosis de $100 \mathrm{mg} / \mathrm{kg}$ aún se observó zonas de necrosis y erosión. Pero a dosis de $400 \mathrm{mg} / \mathrm{kg}$, las zonas de erosión fueron mínimas. Asimismo, a la dosis de $800 \mathrm{mg} / \mathrm{kg}$ se observó en una de las muestras una pequeña erosión superficial pero mínima asociada a anoxia; esto podría deberse a que dicha zona no fue protegida por el EAVT, y dado a que el tiempo de contacto con el etanol solo fue de una hora, a una alta concentración de etanol y por única vez, se trataría de una gastritis aguda.

Dadas las observaciones y mediciones macroscópicas así como la descripción micro anatomo-patológica y en nuestras condiciones experimentales, podemos concluir que el EAVT posee actividad mucoprotectora gástrica en el modelo experimental de lesión gástrica inducida por etanol de $70^{\circ}$ y de manera dosis dependiente. 


\section{AGRADECIMIENTOS}

Al Dr. Javier Arias Stella, director del Instituto de Patología y Biología Molecular Arias-Stella, y al personal del Instituto por las facilidades brindadas en la realización del procesamiento de los cortes histológicos y su posterior diagnóstico microscópico.

\section{REFERENCIAS BIBLIOGRÁFICAS}

1. De la Cruz P. Aprovechamiento integral y racional de la tara Caesalpinia spinosa - Caesalpinia tinctoria. Rev Inst Inv FIGMMG-UNMSM. 2004;7(14):64-73.

2. Aguilar-Galvez A, Noratto G, Chambi F, Debaste F, Campos D. Potential of tara (Caesalpinia spinosa) gallotannins and hydrolysates as natural antibacterial compounds. Food Chem. 2014;156:301-4. doi: 10.1016/j.foodchem.2014.01.110.

3. Avilés R, Carrión J, Huamán J, Bravo M, Rivera D, Rojas N. Actividad antioxidante, polifenoles totales y contenido de taninos de extractos de tara, Caesalpinia spinosa. Rev Per Quim Ing Quím. 2010;13:5-11.

4. López A, Oré R, Miranda C, Trabucco J, Orihuela D, Linares J, y col. Capacidad antioxidante de poblaciones silvestres de "tara" (Caesalpinia spinosa) de las localidades de Picoy y Santa Fe (Provincia de Tarma, departamento de Junin). Sci Agropecu. 2011;2:25-9.

5. Chia A, Basilio J, Azurin J, Quispe L, Veramendi L, Villa N. Efecto del pre-tratamiento con un extracto de Caesalpinia spinosa ante lesiones en mucosa gástrica inducidas con ácido acetil salicílico en ratas Holtzman. CIMEL. 2013;18(1):30-5.

6. Young V, Kormos W, Chick D, Goroll A. Blueprints Medicine. 5a ed. Philadelphia: Lippincott William \& Wilkins; 2010. 181-2 p.

7. Fernández CJ. Incidencia actual de la gastritis: una breve revisión. Rev CENIC Ciencias Biológicas. 2014;45(1):10-7.

8. González M, Ordóñez A, Feliu J, Zamora P, Espinosa E. Tratado de medicina paliativa y tratamiento de soporte del paciente con cáncer. 2a ed. Madrid: Médica Panamericana. 2007:506-8.

9. Sandoval M, Tenorio J, Tinco A, Loli R, Calderón S. Efecto antioxidante y citoprotector del tocosh Solanum tuberosum "papa" en la mucosa gástrica de animales de experimentación. An Fac med. 2015;76(1):15-20. doi:10.15381/anales. v76i1.11070.

10. Lock O. Investigación Fitoquímica, Métodos en el Estudio de Productos Naturales. Lima: Segunda edición. Editorial Fondo. Pontificia Universidad Católica del Perú; 1994.

11. Oré Sifuentes MR. Efectos hipolipémico y antioxidante de Lepidium meyenii Walp en ratas. (Tesis Doctoral). Lima, Perú: Universidad Nacional Mayor de San Marcos, 2008: 104 pp.

12. Robert A, Nezamis J, Lancaster C, Hanchar A. Cytoprotection by prostaglandins in rats. Prevention of gastric necrosis produced by alcohol, $\mathrm{HCl}, \mathrm{NaOH}$, hypertonic $\mathrm{NaCl}$, and thermal injury. Gastroenterology. 1979;77(3):433-43.

13. Júnior $F$, Oliveira $D$, Augusti $A$, Linde $M$, Paul J, Echeverria G, y col. Protective effects of Croton campestris A. St-Hill in different ulcer models in rodents: Evidence for the involvement of nitric oxide and prostaglandins. J Ethnopharmacol. 2014;153(2):469-77. doi: 10.1016/j. jep.2014.03.005

14. Sandoval M, Huamán O, Oré R, Loli A, Ayala S. Efecto antioxidante y citoprotector del Solanum tuberosum (papa) en la mucosa gástrica de animales de experimentación. An Fac med.
2010;71(3):147-52.

15. Bate-Smith EC. Haemanalysis of tannins: The concept of relative astringency. Phytochemistry. 1973;12:907-12.

16. Calixto M. Plantas medicinales utilizadas en odontologia (Parte I). Kiru. 2006;3(2):80-5.

17. Bussmann R, Malca G, Glenn A, Sharon D, Nilsen $B$, Parris B, y col. Toxicity of medicinal plants used in traditional medicine in Northern Peru. J Ethnopharmacol. 2011;137(1):121-40. doi: 10.1016/j. jep.2011.04.071.

18. Zhang L, Lin $Y$. Tannins from Canarium album with potent antioxidant activity. J Zhejiang Univ Sci B. 2008;9(5):407-15.

19. Gülçin I, Huyut Z, Elmastaş M, Aboul-Enein HY. Radical scavenging and antioxidant activity of tannic acid. Arab J Chem. 2010;3(1):43-53.

20. Valero Y, Colina J, Ineichen E. Efecto del procesamiento sobre la capacidad antioxidante de la ciruela criolla (Prunus domestica). Arch Latinoam Nutr. 2012;62(4):363-9.

21. Gomes de Melo J, De Sousa Araújo T, Thijan Nobre de Almeida V, Lyra de Vasconcelos Cabral D, Do Desterro Rodrigues M, Carneiro do Nascimento S, y col. Antiproliferative activity, antioxidant capacity and tannin content in plants of semi-arid northeastern Brazil. Molecules. 2010;15(12):8534-42.

22. Doroteo VH, Terry C, Díaz C. Compuestos fenólicos y actividades antioxidante, antielastasa , anticolagenasa y fotoprotectora in vitro de Myrciaria dubia (camu camu) y Caesalpinia spinosa (tara) phenolic compounds and antioxidant, antielastase, anticollagenase and photoprotecti. Rev Soc Quim Perú. 2012;78(4):254-63.

23. Flores F, Briones L. Influencia del material parental y zonas de vida en el contenido de taninos en Caesalpinia spinosa L(Mol.) Kuntze. FIAT LUX. 2011;7(1):13-9

24. Melo Ferrari M, Glorio P, Tarazona G. Efecto de la madurez en los componentes de valor comercial (taninos y goma) de tara Caesalpinia spinosa (Molina ) Kuntze. Rev Soc Quím Perú. 2013;79(3):218-28.

25. Akuodor G, Essien A, Chilaka K, Akpan J, Ezeokpo B, Ezeonwumelu J. Gastroprotective effect of the aqueous leaf extract of Guiera senegalensis in albino rats. Asian Pac J Trop Med. 2013;6(10):771-5. doi: 10.1016/S1995-7645(13)60136-4.

26. Vasconcelos $P$, Andreo M, Vilegas W, Hiruma C, Pellizzon C. Effect of Mouriri pusa tannins and flavonoids on prevention and treatment against experimental gastric ulcer. J Ethnopharmacol. 2010;131(1):146-53. doi: 10.1016/j. jep.2010.06.017.

27. Tarnawski A. Mecanismos celulares y moleculares de la mucosa gástrica: La injuria a la mucosa y la acción protectora de los antiácidos. Rev Gastroenterol del Perú. 1995;15(1).

28. Tarnawski A, Ahluwalia A, Jones M. Increased susceptibility of aging gastric mucosa to injury: the mechanisms and clinical implications. World J Gastroenterol. 2014;20(16):4467-82. doi: 10.3748/ wjg.v20.i16.4467

29. Adewoye EO, Salami AT. Anti-ulcerogenic mechanism of magnesium in indomethacin induced gastric ulcer in rats. Niger J Physiol Sci. 2013;28:193-9. 